\title{
Asymptotic Behavior of Positive Solutions of a Competitive System Subject to Environmental Noise
}

\author{
Huili Xiang, ${ }^{1,2}$ Zhuang Fang, ${ }^{1}$ Zuxiong Li, ${ }^{1}$ and Zhijun Liu ${ }^{1,2}$ \\ ${ }^{1}$ Key Laboratory of Biologic Resources Protection and Utilization of Hubei Province, Hubei University for Nationalities, \\ Enshi, Hubei 445000, China \\ ${ }^{2}$ Department of Mathematics, Hubei University for Nationalities, Enshi, Hubei 445000, China
}

Correspondence should be addressed to Zhijun Liu; zhijun_liu47@hotmail.com

Received 28 June 2014; Revised 20 September 2014; Accepted 20 September 2014

Academic Editor: Cengiz Çinar

Copyright (C) 2015 Huili Xiang et al. This is an open access article distributed under the Creative Commons Attribution License, which permits unrestricted use, distribution, and reproduction in any medium, provided the original work is properly cited.

\begin{abstract}
A competitive system subject to environmental noise is established. By using the theory of stochastic differential equations and Lyapunov function, sufficient conditions for the existence, uniqueness, stochastic boundedness, and global attraction of the positive solution of the above system are established, respectively. An example together with its corresponding numerical simulations is presented to confirm our analytical results.
\end{abstract}

\section{Introduction}

Mathematical modelling plays an important role in the mathematical ecology. In the past several years, ecological models based on determinate systems emerged in large numbers (see [1-9]). While the disturbance of environmental noise is unavoidable in the real world, more and more researchers start to pay attention to the study on nonlinear dynamic systems with environmental noise and many valuable results have been obtained (see [10-24]).

In [25], Gopalsamy introduced the following competitive system:

$$
\begin{aligned}
& d x_{1}(t)=x_{1}(t)\left[r_{1}-a_{1} x_{1}(t)-\frac{c_{2} x_{2}(t)}{1+x_{2}(t)}\right] d t, \\
& d x_{2}(t)=x_{2}(t)\left[r_{2}-a_{2} x_{2}(t)-\frac{c_{1} x_{1}(t)}{1+x_{1}(t)}\right] d t,
\end{aligned}
$$

where $x_{i}(t)$ may represent the densities of species. The coefficients $r_{i}, a_{i}, b_{i}$, and $c_{i}$ are all positive constants. In the absence of interspecific interactions, each species is governed by the logistic equation; however, in the presence of interspecific interactions, each species retains the average growth rate of the other. In this contribution, we consider the influence of environmental noise and obtain the following form:

$$
\begin{aligned}
d x_{1}(t)= & x_{1}(t)\left[r_{1}-a_{1} x_{1}(t)-\frac{c_{2} x_{2}(t)}{1+x_{2}(t)}\right] d t \\
& +\sigma_{1} x_{1}(t) d w_{1}(t), \\
d x_{2}(t)= & x_{2}(t)\left[r_{2}-a_{2} x_{2}(t)-\frac{c_{1} x_{1}(t)}{1+x_{1}(t)}\right] d t \\
& +\sigma_{2} x_{2}(t) d w_{2}(t),
\end{aligned}
$$

where $x_{i}(0)>0, d w_{i}(t)$ is independent white noise with $w_{i}(0)=0, t \geq 0$, and $\sigma_{i}^{2}$ represents the intensity of the noise, $i=1,2 . w_{i}(t)$ is standard Brownian motion defined on the complete probability space $\left(\Omega, \mathscr{F},\left\{\mathscr{F}_{t}\right\}_{t \geq 0}, P\right)$ with a filtration $\left\{\mathscr{F}_{t}\right\}_{t \geq 0}$ satisfying the usual conditions (i.e., it is right continuous and $\mathscr{F}_{0}$ contains all $P$-null sets).

In this paper, we focus on the asymptotic behavior of positive solution of system (2). To the best of our knowledge, there are few published papers concerning system (2). The rest of this paper is organized as follows. In Section 2, some preliminaries are introduced. The existence, uniqueness, and stochastic boundedness of positive solution of system (2) are 
discussed in Section 3. The global attraction of system (2) is studied in Section 4. As an application of our main results, we present an example and its numerical simulations to support our theoretical results in Section 5.

\section{Preliminaries}

In this section, we introduce some definitions and lemmas which are useful for establishing our main results.

Definition 1. The solution $\left(x_{1}(t), x_{2}(t)\right)$ of system (2) is stochastically bounded if, for any $\varepsilon_{i} \in(0,1)$, there exist positive constants $H_{i}=H\left(\varepsilon_{i}\right)$ such that

$$
\lim _{t \rightarrow+\infty} \sup P\left\{\left|x_{i}(t)\right|>H_{i}\right\}<\varepsilon_{i}, \quad i=1,2 .
$$

Definition 2. Let $\left(x_{1}(t), x_{2}(t)\right)$ be a positive solution of system (2). If another positive solution $\left(x_{1}^{*}(t), x_{2}^{*}(t)\right)$ of system (2) satisfies

$$
\lim _{t \rightarrow+\infty} E\left(\left|\left(x_{1}(t), x_{2}(t)\right)-\left(x_{1}^{*}(t), x_{2}^{*}(t)\right)\right|\right)=0,
$$

then $\left(x_{1}(t), x_{2}(t)\right)$ is global attractive.

Definition 3. Let $\left(x_{1}(t), x_{2}(t)\right)$ be a positive solution of system (2). The solution $x(t)=\left(x_{1}(t), x_{2}(t)\right)$ to system (2) is said to be exponentially extinct with probability one if

$$
\lim \sup _{t \rightarrow \infty} \frac{\ln x_{i}(t)}{t}<0, \quad \text { a.s. } i=1,2 .
$$

Lemma $4\left(C_{p}\right.$ inequality). Suppose that $a_{1}, a_{2}, \ldots, a_{n}$ are all real numbers; then for any positive real number $p$ we have

$$
\left|a_{1}+a_{2}+\cdots+a_{n}\right|^{p} \leq C_{p}\left(\left|a_{1}\right|^{p}+\left|a_{2}\right|^{p}+\cdots+\left|a_{n}\right|^{p}\right),
$$

where

$$
C_{p}= \begin{cases}1, & 0<p \leq 1, \\ n^{p-1}, & p>1 .\end{cases}
$$

Lemma 5 (see [26]). Let $f(t)$ be a nonnegative integrable and uniformly continuous function defined on $[0,+\infty)$ such that $f(t)$ is integrable and uniformly continuous on $[0,+\infty)$. Then $\lim _{t \rightarrow+\infty} f(t)=0$.

Lemma 6 (see $[27,28]$ ). Suppose that a stochastic process $X(t)$ on $t \geq 0$ satisfies the condition

$$
E|X(t)-X(s)|^{\alpha} \leq c|t-s|^{1+\beta}, \quad 0 \leq s, t<+\infty,
$$

for some positive constants $\alpha, \beta$, and $c$. Then there exists a continuous modification $\widetilde{X}(t)$ of $X(t)$, which has the property that, for every $\gamma \in(0, \beta / \alpha)$, there is a positive random variable $h(\omega)$ such that

$$
\begin{aligned}
& P\left\{\omega: \sup _{0<|t-s|<h(\omega), 0 \leq s, t<+\infty} \frac{|\widetilde{X}(t, \omega)-\widetilde{X}(s, \omega)|}{|t-s|^{\gamma}} \geq \frac{2}{1-2^{-\gamma}}\right\} \\
& \quad=1 .
\end{aligned}
$$

In other words, almost every sample path of $\widetilde{X}$ is locally but uniformly Hölder continuous with exponent $\gamma$.

\section{Existence, Uniqueness, Stochastic Boundedness, and Extinction}

We first present the existence and uniqueness of positive solution of system (2).

Theorem 7. System (2) has a unique positive solution, say $\left(x_{1}(t), x_{2}(t)\right)$, on $t \geq 0$. Furthermore, the solution will remain in $R_{+}^{2}=\left\{\left(x_{1}, x_{2}\right) \in R^{2}: x_{i}>0, i=1,2\right\}$ with probability one.

Proof. The proof of this lemma is rather standard. It is obvious that the coefficients of system (2) are local Lipschitz continuous. Then, for any initial value $\left(x_{1}(0), x_{2}(0)\right)$ with $x_{i}(0)>0$, there exists a unique local solution $\left(x_{1}(t), x_{2}(t)\right), t \in\left[0, \tau_{*}\right)$, where $\tau_{*}$ is the explosion time (see $\left.[10,19]\right)$. Therefore, to prove that the local solution is also global, we only need to show that $\tau_{*}=+\infty$ a.s. Let $n_{0}>0$ be sufficiently large so that every component of $\left(x_{1}(0), x_{2}(0)\right)$ lies in $\left[1 / n_{0}, n_{0}\right]$. For each integer $n \geq n_{0}$, we define the stopping time as follows:

$$
\tau_{n}=\inf \left\{t \in\left[0, \tau_{*}\right): x_{1}(t) \notin\left(\frac{1}{n}, n\right) \text { or } x_{2}(t) \notin\left(\frac{1}{n}, n\right)\right\} \text {. }
$$

Here we set inf $\emptyset=+\infty$ ( $\emptyset$ denotes the empty set). Obviously, $\tau_{n}$ is increasing as $n \rightarrow+\infty$. Denote $\tau_{+\infty}=\lim _{n \rightarrow+\infty} \tau_{n}$, whence $\tau_{+\infty} \leq \tau_{*}$ a.s. We need to show that $\tau_{+\infty}=+\infty$ a.s. Otherwise, there exist constants $T>0$ and $\varepsilon \in(0,1)$ such that $P\left\{\tau_{+\infty} \leq T\right\}>\varepsilon$. Then, by denoting $\Omega_{n}=\left\{\tau_{n} \leq T\right\}$, there exists an integer $n_{1} \geq n_{0}$ such that for all $n \geq n_{1}$,

$$
P\left(\Omega_{n}\right) \geq \varepsilon .
$$

We now define a $C^{2}$-function $V$ as

$$
V\left(x_{1}, x_{2}\right)=\left[x_{1}-\ln x_{1}-1\right]+\left[x_{2}-\ln x_{2}-1\right],
$$

where $x_{1}>0$ and $x_{2}>0$. It is obvious that $V\left(x_{1}, x_{2}\right)$ is nonnegative. By Itô's formula, one has

$$
\begin{aligned}
d V\left(x_{1}(t), x_{2}(t)\right) & \\
= & {\left[\left(r_{1}+a_{1}\right) x_{1}-\frac{c_{2} x_{2}(t)}{1+x_{2}(t)}\left(1-x_{1}(t)\right)\right.} \\
& \quad-a_{1} x_{1}^{2}(t)+\frac{1}{2} \sigma_{1}^{2}-r_{1} \\
& +\left(r_{2}+a_{2}\right) x_{2}-\frac{c_{1} x_{1}(t)}{1+x_{1}(t)}\left(1-x_{2}(t)\right) \\
& \left.\quad-a_{2} x_{2}^{2}(t)+\frac{1}{2} \sigma_{2}^{2}-r_{2}\right] d t \\
+ & \left(1-x_{1}(t)^{-1}\right) \sigma_{1} x_{1}(t) d w_{1}(t) \\
+ & \left(1-x_{2}(t)^{-1}\right) \sigma_{2} x_{2}(t) d w_{2}(t) \\
= & f\left(x_{1}(t), x_{2}(t)\right) d t+\left(1-x_{1}(t)^{-1}\right) \sigma_{1} x_{1}(t) d w_{1}(t) \\
& +\left(1-x_{2}(t)^{-1}\right) \sigma_{2} x_{2}(t) d w_{2}(t),
\end{aligned}
$$


where

$$
\begin{aligned}
f\left(x_{1}, x_{2}\right)= & \left(r_{1}+a_{1}\right) x_{1}+\left(r_{2}+a_{2}\right) x_{2} \\
& -\frac{c_{2} x_{2}}{1+x_{2}}\left(1-x_{1}\right)-\frac{c_{1} x_{1}}{1+x_{1}}\left(1-x_{2}\right) \\
& -a_{1} x_{1}^{2}-a_{2} x_{2}^{2}+\frac{1}{2} \sigma_{1}^{2}+\frac{1}{2} \sigma_{2}^{2}-r_{1}-r_{2} .
\end{aligned}
$$

A calculation can show that $f\left(x_{1}, x_{2}\right)$ is upper bounded, denoted by $H$. Thus (13) can be rewritten as

$$
\begin{aligned}
d V\left(x_{1}(t), x_{2}(t)\right) \leq & H d t+\left(1-x_{1}(t)^{-1}\right) \sigma_{1} x_{1}(t) d w_{1}(t) \\
& +\left(1-x_{2}(t)^{-1}\right) \sigma_{2} x_{2}(t) d w_{2}(t) .
\end{aligned}
$$

Integrating both sides from 0 to $\tau_{n} \wedge T$, we acquire that

$$
\begin{aligned}
& \int_{0}^{\tau_{n} \wedge T} V\left(x_{1}(t), x_{2}(t)\right) \\
& \quad \leq \int_{0}^{\tau_{n} \wedge T}\left[\left(1-x_{1}(t)^{-1}\right) \sigma_{1} x_{1}(t) d w_{1}(t)\right. \\
& \left.\quad+\left(1-x_{2}(t)^{-1}\right) \sigma_{2} x_{2}(t) d w_{2}(t)\right] \\
& \quad+\int_{0}^{\tau_{n} \wedge T} H d t .
\end{aligned}
$$

As a consequence, one has

$$
\begin{aligned}
V\left(x_{1}\left(\tau_{n} \wedge T\right), x_{2}\left(\tau_{n} \wedge T\right)\right)-V\left(x_{1}(0), x_{2}(0)\right) \\
\leq H\left(\tau_{n} \wedge T\right)+\int_{0}^{\tau_{n} \wedge T}\left[\left(1-x_{1}(t)^{-1}\right) \sigma_{1} x_{1}(t) d w_{1}(t)\right. \\
\left.+\left(1-x_{2}(t)^{-1}\right) \sigma_{2} x_{2}(t) d w_{2}(t)\right] .
\end{aligned}
$$

Since $\tau_{n} \wedge T>0$, taking expectations one shows that

$$
\begin{aligned}
& E\left(V\left(x_{1}\left(\tau_{n} \wedge T\right), x_{2}\left(\tau_{n} \wedge T\right)\right)\right) \\
& \quad \leq V\left(x_{1}(0), x_{2}(0)\right)+H E\left(\tau_{n} \wedge T\right) .
\end{aligned}
$$

Thus

$$
E\left(V\left(x_{1}\left(\tau_{n} \wedge T\right), x_{2}\left(\tau_{n} \wedge T\right)\right)\right) \leq V\left(x_{1}(0), x_{2}(0)\right)+H T .
$$

On the other hand, for every $\omega \in \Omega_{n}$, either $x_{1}\left(\tau_{n}\right.$, $\omega)$ or $x_{2}\left(\tau_{n}, \omega\right)$ equals to either $n$ or $1 / n$. Then $V\left(x_{1}\left(\tau_{n}\right.\right.$, $\left.\omega), x_{2}\left(\tau_{n}, \omega\right)\right)$ is not less than either $n-1-\ln n$ or $1 / n-1+\ln n$. Consequently, from (19) we have

$$
\begin{aligned}
& V\left(x_{1}(0), x_{2}(0)\right)+H T \\
& \quad \geq E\left[1_{\Omega_{n}} V\left(x_{1}\left(\tau_{n}, \omega\right), x_{2}\left(\tau_{n}, \omega\right)\right)\right] \\
& \quad \geq \varepsilon\left[(n-1-\ln n) \wedge\left(\frac{1}{n}-1+\ln n\right)\right],
\end{aligned}
$$

where $1_{\Omega_{n}}$ is the indicator function of $\Omega_{n}$. Let $n \rightarrow+\infty$, one can show the following contradiction:

$$
+\infty \leq V\left(x_{1}(0), x_{2}(0)\right)+H T<+\infty .
$$

Hence, $\tau_{+\infty}=+\infty$ a.s. and there exists a unique positive solution $\left(x_{1}(t), x_{2}(t)\right)$ of system (2) on $t \geq 0$. This completes the proof.

Next, we investigate the stochastic boundedness of the positive solutions of system (2). To this end, we first give the following Lemma 8.

Lemma 8. If $x_{i}(0)<r_{i} / a_{i}, i=1,2$, then for any real number $p \geq 1$ the solution $\left(x_{1}(t), x_{2}(t)\right)$ of system (2) satisfies

$$
E\left(x_{i}^{p}(t)\right) \leq K_{i}(p),
$$

where

$$
K_{i}(p)=\left[\frac{r_{i}+((p-1) / 2) \sigma_{i}^{2}}{a_{i}}\right]^{p}, \quad i=1,2 .
$$

Proof. By Itồs formula, one can show that

$$
\begin{aligned}
d x_{i}^{p}(t)= & p x_{i}^{p-1}(t) d x_{i}(t)+\frac{p(p-1)}{2} x_{i}^{p-1}(t) d x_{i}(t) d x_{i}(t) \\
= & \left\{p x_{i}^{p-1}(t) x_{i}(t)\left[r_{i}-a_{i} x_{i}(t)-\frac{c_{j} x_{j}(t)}{1+x_{j}(t)}\right]\right. \\
& \left.+\frac{p(p-1)}{2} \sigma_{i}^{2} x_{i}^{p}(t)\right\} d t \\
& +p \sigma_{i} x_{i}^{p}(t) d w_{i}(t) .
\end{aligned}
$$

Integrating from 0 to $t$, we have

$$
\begin{gathered}
x_{i}^{p}(t)-x_{i}^{p}(0) \\
=\int_{0}^{t} p\left\{x _ { i } ^ { p } ( s ) \left[r_{i}-a_{i} x_{i}(s)-\frac{c_{j} x_{j}(t)}{1+x_{j}(t)}\right.\right. \\
\left.\left.+\frac{(p-1)}{2} \sigma_{i}^{2}\right]\right\} d s \\
+\int_{0}^{t} p \sigma_{i} x_{i}^{p}(t) d w_{i}(t) .
\end{gathered}
$$

Taking expectations, we obtain that

$$
\begin{gathered}
E\left(x_{i}^{p}(t)\right)-E\left(x_{i}^{p}(0)\right) \\
=\int_{0}^{t} p E\left\{x _ { i } ^ { p } ( s ) \left[r_{i}-a_{i} x_{i}(s)-\frac{c_{j} x_{j}(s)}{1+x_{j}(s)}\right.\right. \\
\left.\left.+\frac{(p-1)}{2} \sigma_{i}^{2}\right]\right\} d s .
\end{gathered}
$$


So

$$
\begin{aligned}
& \frac{d E\left(x_{i}^{p}(t)\right)}{d t} \\
& \quad=p E\left\{x_{i}^{p}(t)\left[r_{i}-a_{i} x_{i}(t)-\frac{c_{j} x_{j}(t)}{1+x_{j}(t)}+\frac{(p-1)}{2} \sigma_{i}^{2}\right]\right\} \\
& \leq p r_{i} E\left(x_{i}^{p}(t)\right)-p a_{i} E\left(x_{i}^{p+1}(t)\right)+\frac{(p-1)}{2} \sigma_{i}^{2} E\left(x_{i}^{p}(t)\right) \\
& =p E\left(x_{i}^{p}(t)\right)\left\{\left[r_{i}+\frac{(p-1)}{2} \sigma_{i}^{2}\right]-a_{i}\left[E\left(x_{i}^{p}(t)\right)\right]^{1 / p}\right\} .
\end{aligned}
$$

Let

$$
Z_{i}(t)=E\left(x_{i}^{p}(t)\right)
$$

we have

$$
\frac{d Z_{i}(t)}{d t} \leq p Z_{i}(t)\left[r_{i}+\frac{(p-1)}{2} \sigma_{i}^{2}-a_{i}\left(Z_{i}(t)\right)^{1 / p}\right] .
$$

As a consequence

$$
\frac{d Z_{i}(t)}{Z_{i}(t)} \leq p\left[r_{i}+\frac{(p-1)}{2} \sigma_{i}^{2}-a_{i}\left(Z_{i}(t)\right)^{1 / p}\right] d t .
$$

Noting that $x_{i}(0)<\left(r_{i}+(1 / 2)(p-1) \sigma_{i}^{2}\right) / a_{i}, i=1,2$, we have

$$
0<a_{i}\left(Z_{i}(0)\right)^{1 / p}=a_{i} x_{i}(0)<r_{i}+\frac{1}{2}(p-1) \sigma_{i}^{2} .
$$

Furthermore, using the standard comparison principle, one can show that

$$
\left[E\left(x_{i}(t)\right)^{p}\right]^{1 / p}=\left(Z_{i}(t)\right)^{1 / p} \leq \frac{r_{i}+(1 / 2)(p-1) \sigma_{i}^{2}}{a_{i}} .
$$

Then we can obtain that

$$
E\left(x_{i}^{p}(t)\right) \leq K_{i}(p),
$$

where

$$
K_{i}(p)=\left[\frac{r_{i}+((p-1) / 2) \sigma_{i}^{2}}{a_{i}}\right]^{p} .
$$

This completes the proof.

Finally, we discuss the stochastic boundedness of the positive solutions of system (2).

Theorem 9. If $x_{i}(0)<r_{i} / a_{i}, i=1,2$, then the solution $\left(x_{1}(t), x_{2}(t)\right)$ of system (2) is stochastically bounded.

Proof. On one hand, for any positive number $\delta_{i}, i=1,2$, one derives that

$$
P\left\{\left|x_{i}(t)-E\left(x_{i}(t)\right)\right| \geq \delta_{i}\right\} \geq P\left\{x_{i}(t) \geq E\left(x_{i}(t)\right)+\delta_{i}\right\} .
$$

On the other hand, by the Chebyshev inequality and Lemma 8, we obtain that

$$
\begin{aligned}
& P\left\{\left|x_{i}(t)-E\left(x_{i}(t)\right)\right|>\delta_{i}\right\} \\
& \quad \leq \frac{\operatorname{Var}\left(x_{i}(t)\right)}{\delta_{i}^{2}} \leq \frac{E\left(x_{i}^{2}(t)\right)}{\delta_{i}^{2}} \leq \frac{\left(r_{i}+(1 / 2) \sigma_{i}^{2}\right)^{2}}{a_{i}^{2} \delta_{i}^{2}} .
\end{aligned}
$$

Let $\delta_{i}=\left(r_{i}+(1 / 2) \sigma_{i}^{2}\right) / \sqrt{\varepsilon_{i}} a_{i}$. Then we have

$$
P\left\{x_{i}(t) \geq E\left(x_{i}(t)\right)+\frac{r_{i}+(1 / 2) \sigma_{i}^{2}}{\sqrt{\varepsilon_{i}} a_{i}}\right\} \leq \varepsilon_{i} .
$$

It follows from Lemma 8 that

$$
E\left(x_{i}(t)\right) \leq \frac{r_{i}}{a_{i}}
$$

which, together with (37), leads to

$$
\begin{aligned}
& P\left\{x_{i}(t) \geq \frac{r_{i}}{a_{i}}+\frac{r_{i}+(1 / 2) \sigma_{i}^{2}}{\sqrt{\varepsilon_{i}} a_{i}}\right\} \\
& \quad \leq P\left\{x_{i}(t) \geq E\left(x_{i}(t)\right)+\frac{r_{i}+(1 / 2) \sigma_{i}^{2}}{\sqrt{\varepsilon_{i}} a_{i}}\right\} \leq \varepsilon_{i} .
\end{aligned}
$$

Let $H_{i}\left(\varepsilon_{i}\right)=\left(\left(\sqrt{\varepsilon_{i}}+1\right) r_{i}+(1 / 2) \sigma_{i}^{2}\right) / a_{i} \sqrt{\varepsilon_{i}}$, and noting that $x_{i}(t)>0$, one shows that

$$
P\left\{\left|x_{i}(t)\right| \geq H_{i}\left(\varepsilon_{i}\right)\right\}<\varepsilon_{i} .
$$

Therefore,

$$
\lim _{t \rightarrow \infty} \sup P\left\{\left|x_{i}(t)\right| \geq H_{i}\left(\varepsilon_{i}\right)\right\}<\varepsilon_{i},
$$

which implies that the solution of system (2) is stochastically bounded. The proof is complete.

Theorem 10. Suppose that all coefficients of system (2) are positive and $r_{i}<\sigma_{i}^{2} / 2, i=1,2$. Then the solution $\left(x_{1}(t), x_{2}(t)\right)$ of system (2) is exponentially extinct with probability one.

Proof. Define, respectively, Lyapunov functions $\ln x_{1}(t)$ and $\ln x_{2}(t)$. Then the following conclusions can be obtained by Itô's formula

$$
\begin{aligned}
d\left(\ln x_{1}(t)\right)= & {\left[r_{1}-\frac{1}{2} \sigma_{1}^{2}-a_{1} x_{1}(t)-\frac{c_{2} x_{2}(t)}{1+x_{2}(t)}\right] d t } \\
& +\sigma_{1} d w_{1}(t), \\
d\left(\ln x_{2}(t)\right)= & {\left[r_{2}-\frac{1}{2} \sigma_{2}^{2}-a_{2} x_{1}(t)-\frac{c_{1} x_{1}(t)}{1+x_{1}(t)}\right] d t } \\
& +\sigma_{2} d w_{2}(t) .
\end{aligned}
$$

Integrating from 0 to $t$, one concludes that

$$
\begin{aligned}
& \ln x_{1}(t) \leq \ln x_{1}(0)+\left(r_{1}-\frac{1}{2} \sigma_{1}^{2}\right) t+\int_{0}^{t} \sigma_{1} d w_{1}(s), \\
& \ln x_{2}(t) \leq \ln x_{2}(0)+\left(r_{2}-\frac{1}{2} \sigma_{2}^{2}\right) t+\int_{0}^{t} \sigma_{2} d w_{2}(s) .
\end{aligned}
$$


Dividing $t$ on both sides of (43), sending $t \rightarrow \infty$, and employing the strong law of large numbers for local martingales, one acquires that

$$
\lim \sup _{t \rightarrow \infty} \frac{\ln x_{i}(t)}{t}<0, \quad \text { a.s. } i=1,2 .
$$

This completes the proof.

\section{Global Attraction}

In this section, we first introduce Lemma 11 before we show the global attraction of system (2).

Lemma 11. If $c_{i} \leq a_{i}, x_{i}(0)<r_{i} / a_{i}, i=1,2$, then almost every sample path of the solution $\left(x_{1}(t), x_{2}(t)\right)$ of system (2) is uniformly continuous on $t \geq 0$.

Proof. It follows from system (2) that

$$
\begin{aligned}
x_{1}(t)= & x_{1}(0)+\int_{0}^{t} g_{1}\left(s, x_{1}(s), x_{2}(s)\right) d s \\
& +\int_{0}^{t} h_{1}\left(s, x_{1}(s), x_{2}(s)\right) d w_{1}(s),
\end{aligned}
$$

where

$$
\begin{gathered}
g_{1}\left(s, x_{1}(s), x_{2}(s)\right)=x_{1}(s)\left[r_{1}-a_{1} x_{1}(s)-\frac{c_{2} x_{2}(s)}{1+x_{2}(s)}\right], \\
h_{1}\left(s, x_{1}(s), x_{2}(s)\right)=\sigma_{1} x_{1}(s) .
\end{gathered}
$$

Applying Lemmas 4 and 8 , for any $p>1$, one derives that

$$
\begin{aligned}
E\left(\left|g_{1}\left(s, x_{1}(s), x_{2}(s)\right)\right|^{p}\right) & \\
= & E\left(x_{1}^{p}(s)\left|r_{1}-a_{1} x_{1}(s)-\frac{c_{2} x_{2}(s)}{1+x_{2}(s)}\right|^{p}\right) \\
\leq & \frac{1}{2} E\left(x_{1}^{2 p}(s)\right)+\frac{1}{2} E\left(\left(r_{1}-a_{1} x_{1}(s)-\frac{c_{2} x_{2}(s)}{1+x_{2}(s)}\right)^{2 p}\right) \\
\leq & \frac{1}{2} E\left(x_{1}^{2 p}(s)\right)+\frac{1}{2} E\left[3^{2 p-1}\left|r_{1}\right|^{2 p}+3^{2 p-1}\left|a_{1} x_{1}(s)\right|^{2 p}\right. \\
= & \frac{1}{2} E\left(x_{1}^{2 p}(s)\right)+\frac{1}{2} 3^{2 p-1}\left|r_{1}\right|^{2 p}+\frac{1}{2} 3^{2 p-1}\left|a_{1}\right|^{2 p} E\left(x_{1}^{2 p}(s)\right) \\
& +\frac{1}{2} 3^{2 p-1}\left|c_{2}\right|^{2 p-1} E\left(x_{2}^{2 p}(s)\right) \\
\leq & \frac{1}{2} K_{1}(2 p)+\frac{1}{2} 3^{2 p-1}\left|r_{1}\right|^{2 p}+\frac{1}{2} 3^{2 p-1}\left|a_{1}\right|^{2 p} K_{1}(2 p) \\
& +\frac{1}{2} 3^{2 p-1}\left|c_{2}\right|^{2 p-1} K_{2}(2 p) \triangleq L_{1}(p), \\
E\left(\left|h_{1}\left(s, x_{1}(s), x_{2}(s)\right)\right|^{p}\right) & E\left(\sigma_{1}^{p} x_{1}^{p}(s)\right) \leq \sigma_{1}^{p} E\left(x_{1}^{p}(s)\right) \triangleq M_{1}(p) . \\
= &
\end{aligned}
$$

Without loss of generality, we assume that $p>2$. Using the moment inequality (see [10]) to stochastic integral (45), we can obtain that

$$
\begin{aligned}
E\left|\int_{t_{1}}^{t_{2}} h_{1}\left(s, x_{1}(s), x_{2}(s)\right) d w_{1}(s)\right|^{p} \\
\leqslant\left[\frac{p(p-1)}{2}\right]^{p / 2}\left(t_{2}-t_{1}\right)^{p / 2} \\
\quad \times \int_{t_{1}}^{t_{2}} E\left|h_{1}\left(s, x_{1}(s), x_{2}(s)\right)\right|^{p} d s,
\end{aligned}
$$

where $0 \leqslant t_{1}<t_{2}<+\infty$ and $p>2$. We further let

$$
t_{2}-t_{1}<1, \quad \frac{1}{p}+\frac{1}{q}=1
$$

then by (47), (48), and Lemma 4, one yields that

$$
\begin{aligned}
& E\left|x_{1}\left(t_{2}\right)-x_{1}\left(t_{1}\right)\right|^{p} \\
& =E\left[\int_{t_{1}}^{t_{2}} g_{1}\left(s, x_{1}(s), x_{2}(s)\right) d s\right. \\
& \left.\quad+\int_{t_{1}}^{t_{2}} h_{1}\left(s, x_{1}(s), x_{2}(s)\right) d w_{1}(s)\right]^{p}
\end{aligned}
$$$$
\leq E\left[2^{p-1}\left(\int_{t_{1}}^{t_{2}} g_{1}\left(s, x_{1}(s), x_{2}(s)\right) d s\right)^{p}\right.
$$$$
\left.+2^{p-1}\left(\int_{t_{1}}^{t_{2}} h_{1}\left(s, x_{1}(s), x_{2}(s)\right) d w_{1}(s)\right)^{p}\right]
$$$$
\leq 2^{p-1} E\left(\int_{t_{1}}^{t_{2}}\left|g_{1}\left(s, x_{1}(s), x_{2}(s)\right)\right|^{p} d s\right)
$$$$
+2^{p-1} E\left(\int_{t_{1}}^{t_{2}}\left|h_{1}\left(s, x_{1}(s), x_{2}(s)\right)\right|^{p} d w_{1}(s)\right)
$$$$
\leq 2^{p-1}\left(\int_{t_{1}}^{t_{2}} 1^{q} d s\right)^{p / q} E\left(\int_{t_{1}}^{t_{2}}\left|g_{1}\left(s, x_{1}(s), x_{2}(s)\right)\right|^{p} d s\right)
$$$$
+2^{p-1}\left[\frac{p(p-1)}{2}\right]^{p / 2}\left(t_{2}-t_{1}\right)^{(p-2) / 2}
$$$$
\times \int_{t_{1}}^{t_{2}} E\left|h_{1}\left(s, x_{1}(s), x_{2}(s)\right)\right|^{p} d s
$$$$
\leq 2^{p-1}\left(\int_{t_{1}}^{t_{2}} 1^{q} d s\right)^{p / q} E\left(\int_{t_{1}}^{t_{2}} L_{1}(p) d s\right)
$$$$
+2^{p-1}\left[\frac{p(p-1)}{2}\right]^{p / 2}\left(t_{2}-t_{1}\right)^{(p-2) / 2} \int_{t_{1}}^{t_{2}} M_{1}(p) d s
$$$$
=2^{p-1} L_{1}(p)\left(t_{2}-t_{1}\right)^{p / 2}
$$ 


$$
\begin{aligned}
& +2^{p-1}\left[\frac{p(p-1)}{2}\right]^{p / 2}\left(t_{2}-t_{1}\right)^{(p-2) / 2} M_{1}(p) \\
\leq & 2^{p-1}\left(t_{2}-t_{1}\right)^{(p-2) / 2}\left[L_{1}(p)+\left[\frac{p(p-1)}{2}\right]^{p / 2} M_{1}(p)\right] .
\end{aligned}
$$

It follows from Lemma 6 that almost every sample path of $x_{1}(t)$ is uniformly continuous on $t \geq 0$. Similarly, we can show that almost every sample path of $x_{2}(t)$ is uniformly continuous on $t \geq 0$. Therefore, $\left(x_{1}(t), x_{2}(t)\right)$ is uniformly continuous on $t \geq 0$, a.s. This completes the proof.

We can now present the result on global attraction of system (2).

Theorem 12. If $c_{i} \leq a_{i}, x_{i}(0)<r_{i} / a_{i}, i=1,2$, then system (2) has a unique global attractive positive solution, denoted by $\left(x_{1}(t), x_{2}(t)\right)$, on $t \geq 0$.

Proof. It follows from Theorem 7 that system (2) has a unique positive solution $\left(x_{1}(t), x_{2}(t)\right)$. Assume that $\left(x_{1}^{*}(t), x_{2}^{*}(t)\right)$ is another positive solution of system (2). Consider a Lyapunov function $V(t)$ defined by

$$
V(t)=\left|\ln x_{1}(t)-\ln x_{1}^{*}(t)\right|+\left|\ln x_{2}(t)-\ln x_{2}^{*}(t)\right|, \quad t \geq 0 .
$$

Applying Itô's formula, a calculation of the right differential $D^{+} V(t)$ of $V(t)$ along the solution, one yields that

$$
\begin{aligned}
& D^{+} V(t) \\
& =\operatorname{sgn}\left(x_{1}(t)-x_{1}^{*}(t)\right)\left\{\left[\frac{d x_{1}(t)}{x_{1}(t)}-\frac{\left(d x_{1}(t)\right)^{2}}{2 x_{1}^{2}(t)}\right]\right. \\
& \left.-\left[\frac{d x_{1}^{*}(t)}{x_{1}^{*}(t)}-\frac{\left(d x_{1}^{*}(t)\right)^{2}}{2 x_{1}^{*}(t)^{2}}\right]\right\} \\
& +\operatorname{sgn}\left(x_{2}(t)-x_{2}^{*}(t)\right)\left\{\left[\frac{d x_{2}(t)}{x_{2}(t)}-\frac{\left(d x_{2}(t)\right)^{2}}{2 x_{2}^{2}(t)}\right]\right. \\
& \left.-\left[\frac{d x_{2}^{*}(t)}{x_{2}^{*}(t)}-\frac{\left(d x_{2}^{*}(t)\right)^{2}}{2 x_{2}^{*}(t)^{2}}\right]\right\} \\
& =\operatorname{sgn}\left(x_{1}(t)-x_{1}^{*}(t)\right) \\
& \times\left\{\left[\left(r_{1}-a_{1} x_{1}(t)-\frac{c_{2} x_{2}(t)}{1+x_{2}(t)}-\frac{\sigma_{1}^{2}}{2}\right) d t\right.\right. \\
& +\sigma_{1} d w_{1}(t) \\
& -\left[\left(r_{1}-a_{1} x_{1}^{*}(t)-\frac{c_{2} x_{2}^{*}(t)}{1+x_{2}^{*}(t)}-\frac{\sigma_{1}^{2}}{2}\right) d t\right. \\
& \left.\left.+\sigma_{1} d w_{1}(t)\right]\right\}
\end{aligned}
$$

$$
\begin{aligned}
& +\operatorname{sgn}\left(x_{2}(t)-x_{2}^{*}(t)\right) \\
& \times\left\{\left[\left(r_{2}-a_{2} x_{2}(t)-\frac{c_{1} x_{1}(t)}{1+x_{1}(t)}-\frac{\sigma_{2}^{2}}{2}\right) d t\right.\right. \\
& \left.+\sigma_{2} d w_{2}(t)\right] \\
& \quad-\left[\left(r_{2}-a_{2} x_{2}^{*}(t)-\frac{c_{1} x_{1}^{*}(t)}{1+x_{1}^{*}(t)}-\frac{\sigma_{2}^{2}}{2}\right) d t\right. \\
& +\operatorname{sgn}\left(x_{1}(t)-x_{1}^{*}(t)\right) \\
& \times\left[a_{1}\left(x_{1}^{*}(t)-x_{1}(t)\right)\right. \\
& \left.+c_{1}\left(\frac{x_{1}^{*}(t)}{1+x_{1}^{*}(t)}-\frac{x_{1}(t)}{1+x_{1}(t)}\right)\right] d t . \\
& +\operatorname{sgn}\left(x_{2}(t)-x_{2}^{*}(t)\right) \\
& \times\left[a_{2}\left(x_{2}^{*}(t)-x_{2}(t)\right)\right. \\
& +x_{2}^{*}(t) \\
& \left.\left.+\frac{x_{2}(t)}{1+x_{2}(t)}\right)\right] d t
\end{aligned}
$$

Integrating from 0 to $t$ and taking expectations one can show that

$$
\begin{aligned}
& E(V(t)-V(0)) \\
& =E\left\{\int_{0}^{t} \operatorname{sgn}\left(x_{1}(s)-x_{1}^{*}(s)\right)\right. \\
& \quad \times\left[a_{1}\left(x_{1}^{*}(s)-x_{1}(s)\right)\right. \\
& \left.\quad+c_{2}\left(\frac{x_{2}^{*}(s)}{1+x_{2}^{*}(s)}-\frac{x_{2}(s)}{1+x_{2}(s)}\right)\right] d s \\
& +\int_{0}^{t} \operatorname{sgn}\left(x_{2}(s)-x_{2}^{*}(s)\right) \\
& \quad \times\left[a_{2}\left(x_{2}^{*}(s)-x_{2}(s)\right)\right. \\
& \left.\left.\quad+c_{1}\left(\frac{x_{1}^{*}(s)}{1+x_{1}^{*}(s)}-\frac{x_{1}(s)}{1+x_{1}(s)}\right)\right] d s\right\} .
\end{aligned}
$$

Thus

$$
\begin{aligned}
& \frac{d E(V(t))}{d t} \\
& =E\left\{\operatorname{sgn}\left(x_{1}(t)-x_{1}^{*}(t)\right)\right.
\end{aligned}
$$




$$
\begin{gathered}
\times\left[a_{1}\left(x_{1}^{*}(t)-x_{1}(t)\right)\right. \\
\left.\quad+c_{2}\left(\frac{x_{2}^{*}(t)}{1+x_{2}^{*}(t)}-\frac{x_{2}(t)}{1+x_{2}(t)}\right)\right] \\
+\operatorname{sgn}\left(x_{2}(t)-x_{2}^{*}(t)\right) \\
\times\left[a_{2}\left(x_{2}^{*}(t)-x_{2}(t)\right)\right. \\
\left.\left.\quad+c_{1}\left(\frac{x_{1}^{*}(t)}{1+x_{1}^{*}(t)}-\frac{x_{1}(t)}{1+x_{1}(t)}\right)\right]\right\} \\
\leq-a_{1} E\left(\left|x_{1}(t)-x_{1}^{*}(t)\right|\right)+c_{2} E\left(\left|x_{2}(t)-x_{2}^{*}(t)\right|\right) \\
-a_{2} E\left(\left|x_{2}(t)-x_{2}^{*}(t)\right|\right)+c_{1} E\left(\left|x_{1}(t)-x_{1}^{*}(t)\right|\right) \\
=\left(c_{1}-a_{1}\right) E\left(\left|x_{1}(t)-x_{1}^{*}(t)\right|\right) \\
+\left(c_{2}-a_{2}\right) E\left(\left|x_{2}(t)-x_{2}^{*}(t)\right|\right),
\end{gathered}
$$

and hence integrating from 0 to $t$ one derives that

$$
\begin{aligned}
E(V(t)) \leq & \int_{0}^{t}\left(c_{1}-a_{1}\right) E\left(\left|x_{1}(s)-x_{1}^{*}(s)\right|\right) d s \\
& +\int_{0}^{t}\left(c_{2}-a_{2}\right) E\left(\left|x_{2}(s)-x_{2}^{*}(s)\right|\right) d s+V(0),
\end{aligned}
$$

which implies that

$$
\begin{aligned}
E\left(\left|\left(x_{1}(t), x_{2}(t)\right)-\left(x_{1}^{*}(t), x_{2}^{*}(t)\right)\right|\right) \\
=E\left\{\left[\left|x_{1}(t)-x_{1}^{*}(t)\right|^{2}+\left|x_{2}(t)-x_{2}^{*}(t)\right|^{2}\right]^{1 / 2}\right\} \\
\leq E\left(\left|x_{1}(t)-x_{1}^{*}(t)\right|\right) \\
\quad+E\left(\left|x_{2}(t)-x_{2}^{*}(t)\right|\right) \in L^{1}[0,+\infty) .
\end{aligned}
$$

So

$$
\begin{aligned}
E & \left(\left|\left(x_{1}(t), x_{2}(t)\right)-\left(x_{1}^{*}(t), x_{2}^{*}(t)\right)\right|\right) \\
= & E\left\{\left[\left|x_{1}(t)-x_{1}^{*}(t)\right|^{2}+\left|x_{2}(t)-x_{2}^{*}(t)\right|^{2}\right]^{1 / 2}\right\} \\
\leq & E\left(\left|x_{1}(t)-x_{1}^{*}(t)\right|\right) \\
& +E\left(\left|x_{2}(t)-x_{2}^{*}(t)\right|\right) \in L^{1}[0, \infty),
\end{aligned}
$$

which, together with Lemmas 5 and 11, leads to

$$
\lim _{t \rightarrow \infty} E\left(\left|\left(x_{1}(t), x_{2}(t)\right)-\left(x_{1}^{*}(t), x_{2}^{*}(t)\right)\right|\right)=0
$$

and hence $\left(x_{1}(t), x_{2}(t)\right)$ is global attractive on $t \geq 0$.

\section{An Example}

In this section, we first give an example to verify the feasibilities of Theorems 9 and 12. Using the Milsten method

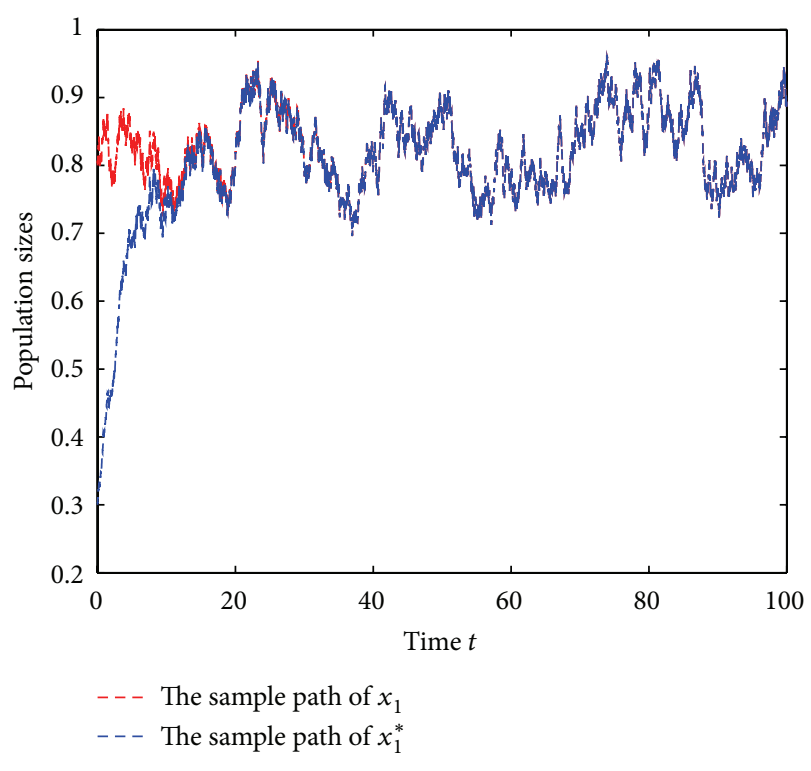

FIGURE 1: The sample paths of $x_{1}(t)$ and $x_{1}^{*}(t)$.

mentioned in Higham [29], we can derive the following discrete version of system (2):

$$
\begin{aligned}
x_{1}(k+1)-x_{1}(k) \\
=x_{1}(k)\left[r_{1}-a_{1} x_{1}(k)-\frac{c_{2} x_{2}(k)}{1+x_{2}(k)}\right] \Delta t \\
\quad+\sigma_{1} x_{1}(k) \sqrt{\Delta t} N_{1}(k) \\
\quad+\frac{1}{2} \sigma_{1}^{2} x_{1}(k)\left(N_{1}^{2}(k)-1\right) \Delta t, \\
x_{2}(k+1)-x_{2}(k) \\
=x_{2}(k)\left[r_{2}-a_{2} x_{2}(k)-\frac{c_{1} x_{1}(k)}{1+x_{1}(k)}\right] \Delta t \\
\quad+\sigma_{2} x_{2}(k) \sqrt{\Delta t} N_{2}(k) \\
+\frac{1}{2} \sigma_{2}^{2} x_{2}(k)\left(N_{2}^{2}(k)-1\right) \Delta t,
\end{aligned}
$$

where $N_{1}(k)$ and $N_{2}(k)$ are Gaussian random variables which follow $N(0,1)$. Let us choose $r_{1}=0.5, a_{1}=0.4, c_{1}=0.1$, $\sigma_{1}=0.1, r_{2}=0.6, a_{2}=0.5, c_{2}=0.3, \sigma_{2}=0.1, \Delta t=0.001$, and $\left(x_{1}(0), x_{2}(0)\right)=(0.8,1.1),\left(x_{1}^{*}(0), x_{2}^{*}(0)\right)=(0.3,0.4)$. $\mathrm{A}$ calculation shows that the conditions of Theorems 9 and 12 are satisfied. Figures 1 and 2 show that the positive solution of system (59) is stochastically bounded and global attractive on $t \geq 0$.

Recalling the whole paper, we have derived sufficient conditions for the existence, uniqueness, stochastic boundedness, and global attraction of the positive solutions of system (2). However, there are still some limitations in our work which need to be improved. We only especially consider the white noise which is an idealized situation. In fact, the effect 


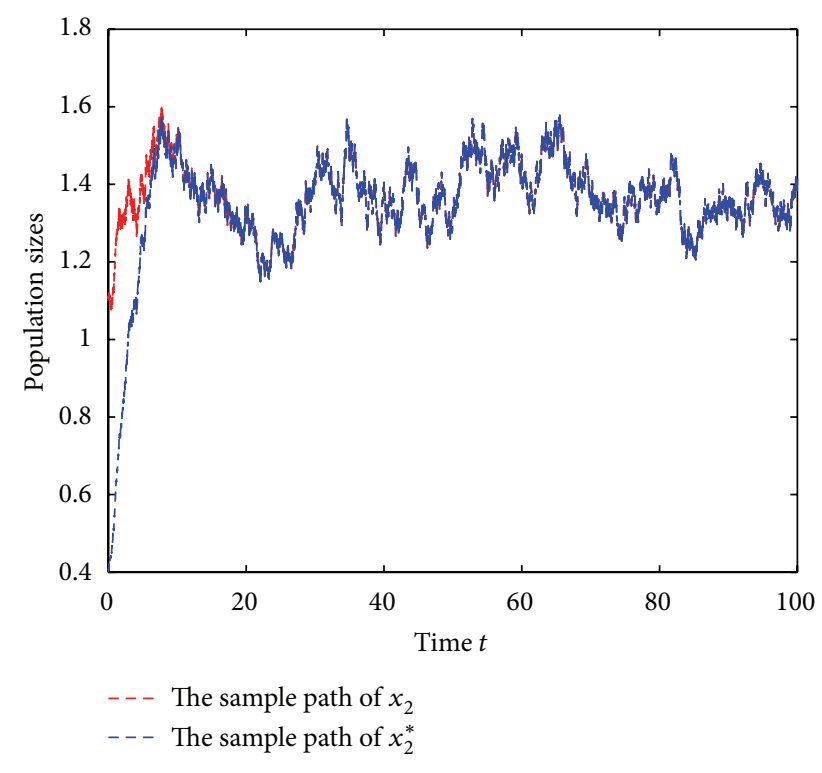

FIGURE 2: The sample paths of $x_{2}(t)$ and $x_{2}^{*}(t)$.

of colorful noise on system (2) is more general in line with the actual situation, and we leave it for our future work.

\section{Conflict of Interests}

The authors declare that there is no conflict of interests regarding the publication of this paper.

\section{Acknowledgments}

The work is supported by the National Natural Science Foundation of China (no. 11261017), the Key Project of Chinese Ministry of Education (no. 212111), the Key Laboratory of Biological Resources Protection and Utilization of Hubei Province (PKLHB1323), and the Key Subject of Hubei Province (Mathematics, Forestry), and the Project of Education Department of Hubei Province (B20111909).

\section{References}

[1] X. Tang, D. Cao, and X. Zou, "Global attractivity of positive periodic solution to periodic Lotka-Volterra competition systems with pure delay," Journal of Differential Equations, vol. 228, no. 2, pp. 580-610, 2006.

[2] R. Q. Shi, X. W. Jiang, and L. S. Chen, "A predator-prey model with disease in the prey and two impulses for integrated pest management," Applied Mathematical Modelling, vol. 33, no. 5, pp. 2248-2256, 2009.

[3] Z. Li, M. Han, and F. Chen, "Influence of feedback controls on an autonomous Lotka-Volterra competitive system with infinite delays," Nonlinear Analysis: Real World Applications, vol. 14, no. 1, pp. 402-413, 2013.

[4] J. Jiao and L. Chen, "The genic mutation on dynamics of a predator-prey system with impulsive effect," Nonlinear Dynamics, vol. 70, no. 1, pp. 141-153, 2012.
[5] C. Wei and L. Chen, "Heteroclinic bifurcations of a preypredator fishery model with impulsive harvesting," International Journal of Biomathematics, vol. 6, no. 5, Article ID 1350031, 2013.

[6] K. Gopalsamy, "Stability of artificial neural networks with impulses," Applied Mathematics and Computation, vol. 154, no. 3, pp. 783-813, 2004.

[7] H.-C. Chen and C.-P. Ho, "Persistence and global stability on competition system with time-delay," Tunghai Science, no. 5, pp. 71-99, 2003.

[8] Z. Liu, J. Wu, and R. A. Cheke, "Coexistence and partial extinction in a delay competitive system subject to impulsive harvesting and stocking," IMA Journal of Applied Mathematics, vol. 75, no. 5, pp. 777-795, 2010.

[9] Z. J. Liu and Q. L. Wang, "An almost periodic competitive system subject to impulsive perturbations," Applied Mathematics and Computation, vol. 231, pp. 377-385, 2014.

[10] A. Friedman, Stochastic Differential Equations and their Applications, Academic Press, 1976.

[11] T. C. Gard, Introduction to Stochastic Differential Equations, Marcel Dekker, New York, NY, USA, 1988.

[12] O. Kallenberg, Foundations of Modern Probability, Springer, Berlin, Germany, 1997.

[13] X. R. Mao, G. Marion, and E. Renshaw, "Environmental Brownian noise suppresses explosions in population dynamics," Stochastic Processes and their Applications, vol. 97, no. 1, pp. 95110, 2002.

[14] R. Z. Khasminskii and F. C. Klebaner, "Long term behavior of solutions of the Lotka-Volterra system under small random perturbations," The Annals of Applied Probability, vol. 11, no. 3, pp. 952-963, 2001.

[15] Y. Li and H. Gao, "Existence, uniqueness and global asymptotic stability of positive solutions of a predator-prey system with Holling II functional response with random perturbation," Nonlinear Analysis: Theory, Methods \& Applications, vol. 68, no. 6, pp. 1694-1705, 2008.

[16] M. Liu and K. Wang, "Dynamics of a two-prey one-predator system in random environments," Journal of Nonlinear Science, vol. 23, no. 5, pp. 751-775, 2013.

[17] X.-Z. Meng, "Stability of a novel stochastic epidemic model with double epidemic hypothesis," Applied Mathematics and Computation, vol. 217, no. 2, pp. 506-515, 2010.

[18] S. Li and X. A. Zhang, "Qualitative analysis of a stochastic predator-prey system with disease in the predator," International Journal of Biomathematics, vol. 6, no. 1, Article ID 1250068, 13 pages, 2013.

[19] L. Arnold, Stochastic Differential Equations: Theory and Applications, Wiley, New York, NY, USA, 1972.

[20] X. Li and X. Mao, "Population dynamical behavior of nonautonomous Lotka-Volterra competitive system with random perturbation," Discrete and Continuous Dynamical Systems, vol. 24, no. 2, pp. 523-545, 2009.

[21] Y. Xu, F. K. Wu, and Y. M. Tan, "Stochastic Lotka-Volterra system with infinite delay," Journal of Computational and Applied Mathematics, vol. 232, no. 2, pp. 472-480, 2009.

[22] D. Q. Jiang, C. Y. Ji, X. Y. Li, and D. O'Regan, "Analysis of autonomous Lotka-Volterra competition systems with random perturbation," Journal of Mathematical Analysis and Applications, vol. 390, no. 2, pp. 582-595, 2012.

[23] Y. Huang, Q. Liu, and Y. Liu, "Global asymptotic stability of a general stochastic Lotka-Volterra system with delays," Applied Mathematics Letters, vol. 26, no. 1, pp. 175-178, 2013. 
[24] Y. Hu, F. Wu, and C. Huang, "Stochastic Lotka-Volterra models with multiple delays," Journal of Mathematical Analysis and Applications, vol. 375, no. 1, pp. 42-57, 2011.

[25] K. Gopalsamy, Stability and Oscillations in Delay Differential Equations of Population Dynamics, Kluwer Academic, Dordrecht, The Netherlands, 1992.

[26] I. Barbalat, "Systems dequations differentielles d'osci d'oscillations nonlinears," Revue Roumaine de Mathematiques Pures et Appliquees, vol. 4, no. 2, pp. 267-270, 1959.

[27] X. R. Mao, "Stochastic versions of the LaSalle theorem," Journal of Differential Equations, vol. 153, no. 1, pp. 175-195, 1999.

[28] I. Karatzas and S. E. Shreve, Brownian Motion and Stochastic Calculus, vol. 113 of Graduate Texts in Mathematics, Springer, New York, NY, USA, Second edition, 1991.

[29] D. J. Higham, "An algorithmic introduction to numerical simulation of stochastic differential equations," SIAM Review, vol. 43, no. 3, pp. 525-546, 2001. 


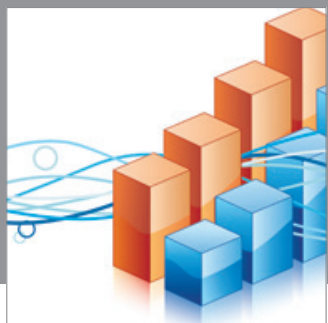

Advances in

Operations Research

mansans

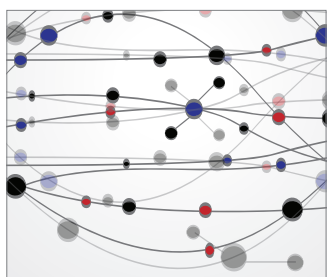

The Scientific World Journal
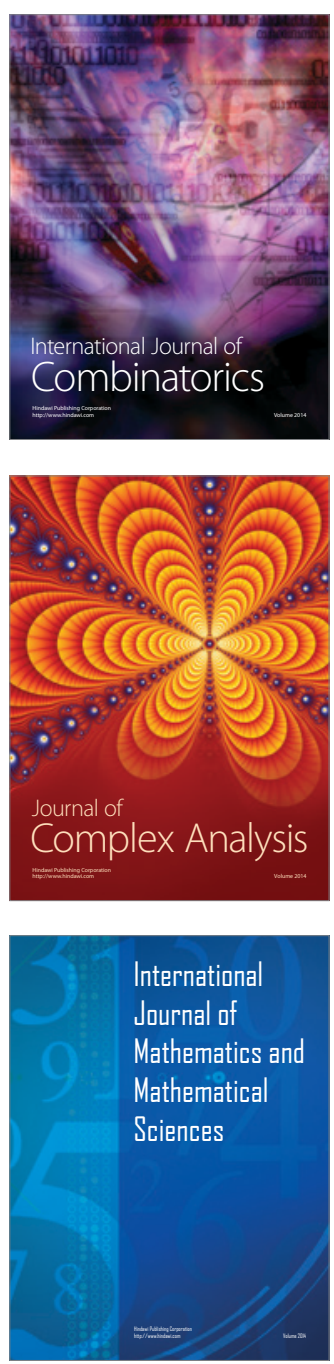
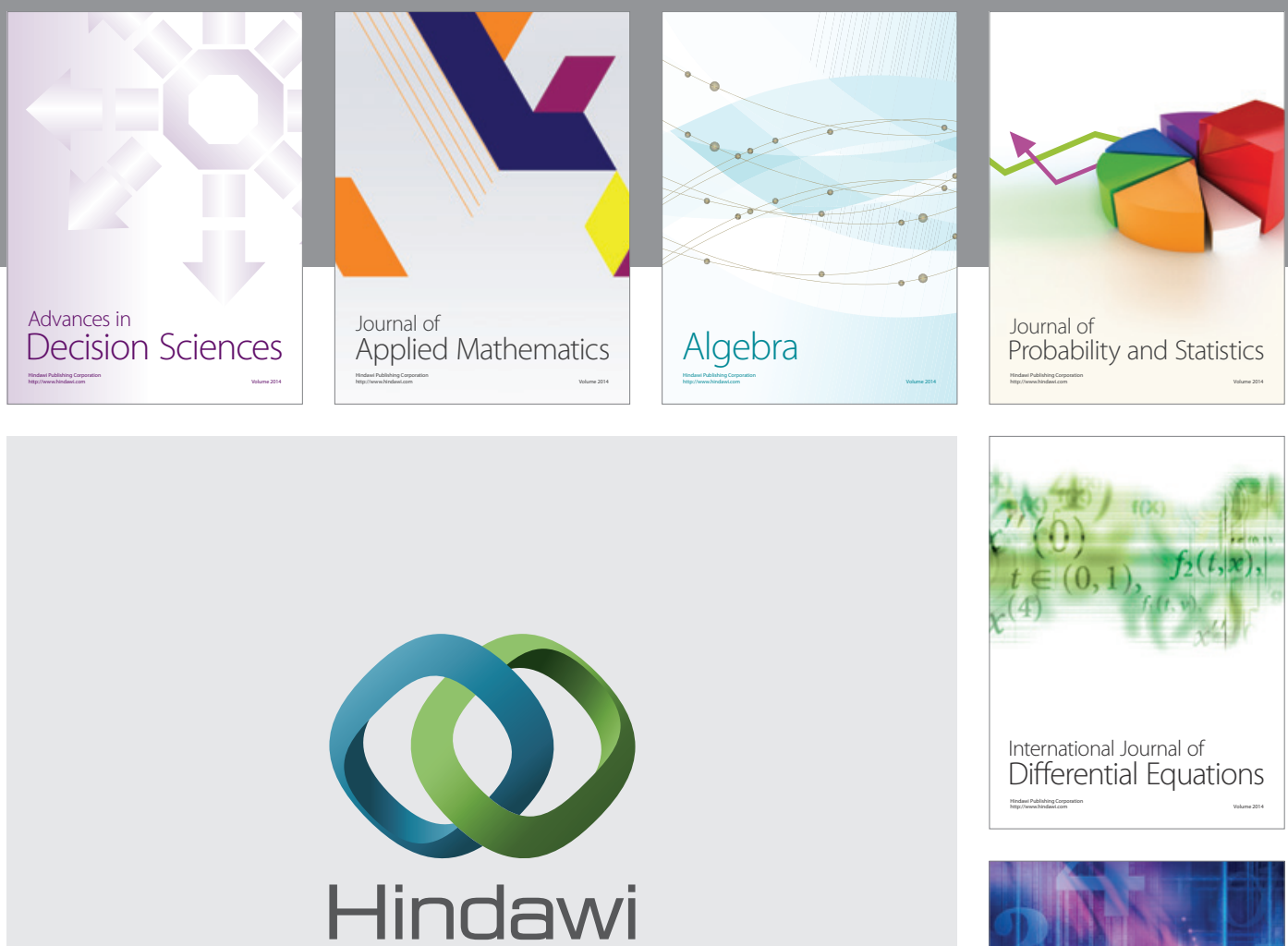

Submit your manuscripts at http://www.hindawi.com
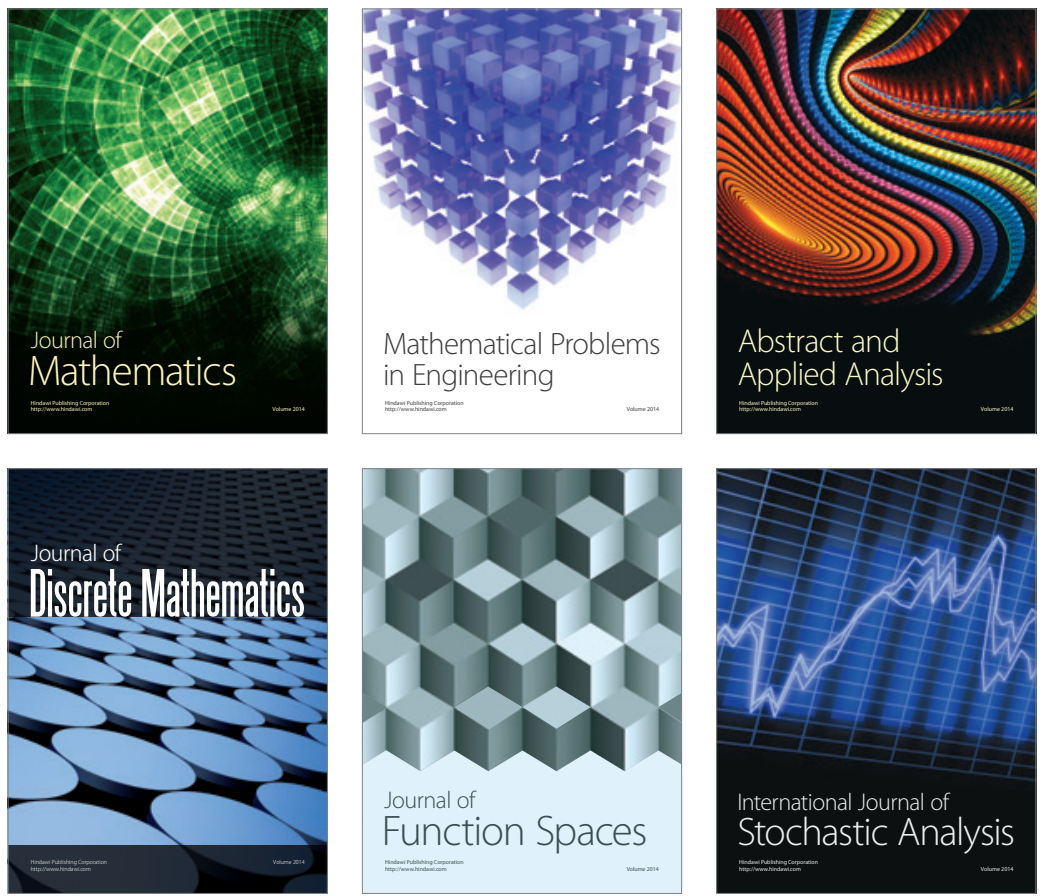

Journal of

Function Spaces

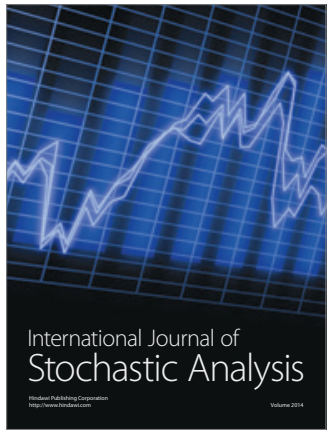

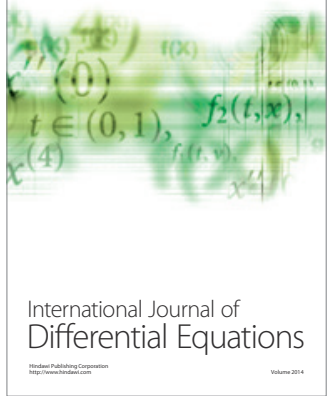
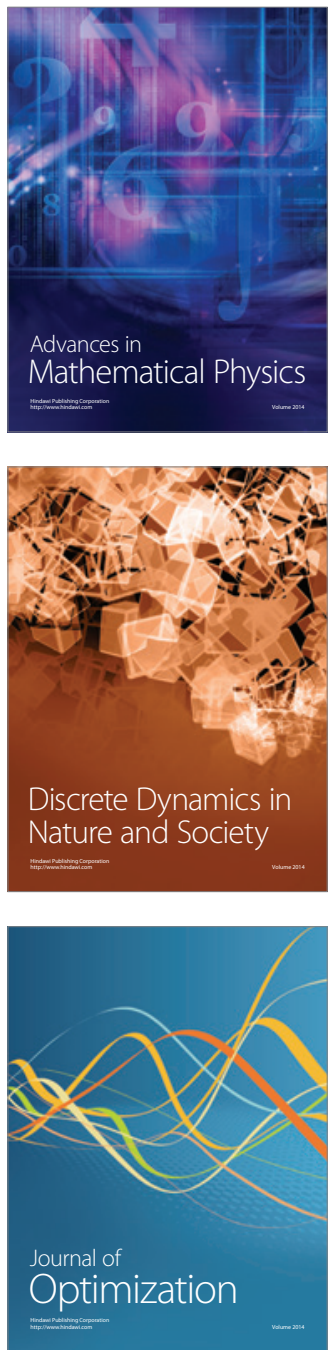